\title{
Probabilistic assessment of the impact of material properties on the risk of frost damage in masonry constructions
}

\author{
Klaas Calle ${ }^{1, *}$, and Nathan Van Den Bossche ${ }^{1}$ \\ ${ }^{1}$ Faculty of Eng. and Arch., Building Physics group, Ghent University, 9000 Ghent Belgium
}

\begin{abstract}
Historically valuable buildings often contain a huge energy saving potential due to their current lacking thermal performance of the building envelope. Next to this, unfortunately insulation measures, which normally occur at the inside, induce a decrease in the temperature and drying potential of the structure which results in a risk for frost damage. To validate this risk Heat, Air and Moisture simulations are a valuable tool. Heritage facades often consist of masonries, which are known to have a complex hygrothermal behaviour. Therefore nowadays a homogenization of the masonry, for example to a homogenous brick wall, is often assumed to save computational time. Nevertheless these homogenizations, which include neglecting mortars, can have a drastic impact on the hygrothermal behaviour of masonries. Hence this study compares the impact of basic material parameters for the brick, pointing mortar and bed mortar of a masonry separately under a constant indoor and outdoor climate based on probabilistic simulations. The evaluation is done for three frost related criteria. Generally, the main impact parameters for the one and a half brick construction are found to be quite similar for interior insulated and not insulated cases but diverse between the chosen criteria.
\end{abstract}

\section{Introduction}

Probabilistic analyses are highly valuable to investigate the dominant impact parameters for certain damage criteria. For example, during the renovation process of historically valuable brickwork facades with interior insulation, the question of risk inducement related to frost damage is quickly asked. Based on the basic principles behind frost damage [1] it is clear that both the moisture content and the temperature play an important role. But the impact of variations of the moisture storage, moisture transport and thermal properties of the materials in a specific construction cannot be quantified by intuition.

This paper investigates the dominant impact parameters on material level of a one and a half brick masonry construction (un)insulated cases.

One of the main challenges in probabilistic risk assessment with Heat, Air and Moisture(HAM) simulation is the computational time. Due to recent developments in the calculation power of computers, advanced sampling techniques and high performance

*Corresponding author: klaas.calle@gent.be 
solvers this can be partially overcome even for $2 \mathrm{D}$ construction, which allows us to implement a pointing mortar(PM), a bed mortar(BM) and brick(B) in the simulated section of a masonry construction.

\section{Construction}

As introduced, a 2D section of a masonry construction is simulated(Fig. 1.). The section includes a pointing mortar, a bed mortar and two bricks. At the inside a plaster is present but during sampling $(\S 3)$ the material properties of this layer are kept constant. HAM simulation software, Delphin 5.9, is used as a detailed ice model is implemented. Between the interior plaster and the masonry interface a thermal/vapour resistance $\left(\mathrm{R} / \mathrm{S}_{\mathrm{d}}\right)$ of respectively $4\left[\mathrm{~m}^{2} \mathrm{~K} / \mathrm{W}\right]$ and $100[-]$ is applied. The exterior climate of Essen and an interior climate defined based on EN ISO 13788 [2] are used as boundary conditions.

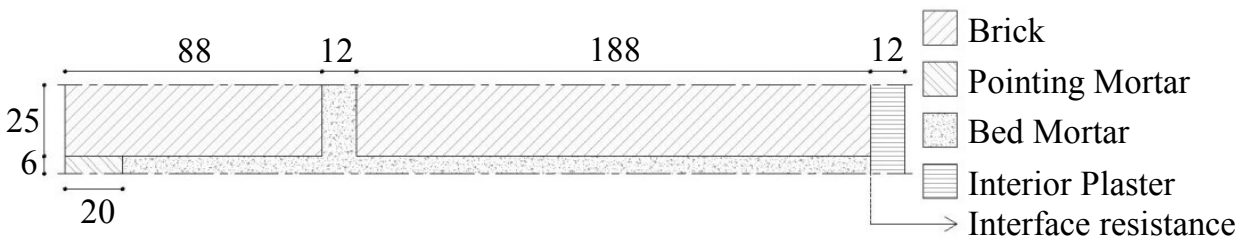

Fig. 1. Abstraction of the simulated construction [mm]

\section{Sampling}

The sampling is executed based on uniform Latin Hypercube sampling as this is proven in literature to be an efficient sampling method for hygrothermal simulations of buildings [3][6]. For each of the three variable materials present in the construction seven material parameters are sampled respectively: density, heat capacity, thermal conductivity, vapour conductivity, porosity and liquid conductivity and moisture content at effective saturation which results in a sampling with 21 variable input parameters. The Technical University of Dresden(TUD) defined, in perspective of Annex 55, Subtask 1: Stochastic data [4], the distribution type of these parameters and defined the mean and standard deviation of the parameters for several building material groups. For this study the data of the groups 'bricks' and 'plasters and mortars' are used respectively for the brickwork and the pointing/bed mortar (Table. 1).

Zhao J. intensively studied the statistics behind building material characterizations in his dissertation. In context of this study he computed the correlation between the first five material properties summed up above [3]. As it is clear that for example a high density material will most likely also have a higher thermal conductivity and a lower porosity, the derived correlations by Zhoa J. are used to ensure the reliability of the sampling (Table 2) [3]. The material functions: moisture retention, liquid conductivity and vapour conductivity curve are scaled based on the sampled material properties. The trends of the curves on the other hand are based on a cluster brick defined by Zhao J. (ID97,TUD-database) for the brick and for the mortars a standard lime cement mortar (ID 718, TUD-database) is used.

During sampling, samples in the tails of the distributions which are found to be impossible on physical grounds are disregarded, the rejection criteria in the last column of Table 1 are applied. This method is different than the common method of sampling material instead of material properties. The benefit of the sampling of material properties is that the amount of samples can easily be increased and further refined. 
Table 1. Material parameters [3]

\begin{tabular}{c|cccc}
$\begin{array}{c}\text { Basic } \\
\text { parameters }\end{array}$ & Distribution & Brick & $\begin{array}{c}\text { Pointing/Bed } \\
\text { mortar }\end{array}$ & $\begin{array}{c}\text { Rejection } \\
\text { criteria }\end{array}$ \\
\hline$\rho\left[\mathrm{kg} / \mathrm{m}^{3}\right]$ & normal & $1791(216)$ & $1357(365)$ & $>0$ \\
$\mathrm{CE}[\mathrm{J} / \mathrm{Kg} \cdot \mathrm{K}]$ & normal & $867(65)$ & $949(234)$ & $>0$ \\
$\lambda\left[\mathrm{W} / \mathrm{m}^{2} \mathrm{~K}\right]$ & normal & $0.684(0.218)$ & $0.454(0.221)$ & $>0$ \\
$\mu[-]^{*}$ & lognormal & $3.031(0.599)$ & $2.617(0.393)$ & $>0,<100$ \\
$\vartheta \_p o r\left[\mathrm{~m}^{3} / \mathrm{m}^{3}\right]$ & normal & $0.313(0.063)$ & $0.501(0.136)$ & $>0,<1$ \\
Log10 $(\mathrm{KI}$ eff) $[\mathrm{s}]$ & normal & $-8.44(0.84)$ & $-9.98(1.1)$ & $/$ \\
$\vartheta \_e f f\left[\mathrm{~m}^{3} / \mathrm{m}^{3}\right]$ & normal & $0.285(0.07)$ & $0.31(0.083)$ & $>0,<\vartheta \_p o r$
\end{tabular}

Table 2. Correlation matrices [3]

\begin{tabular}{c|ccccc} 
& $\boldsymbol{\rho}$ & $\mathbf{C E}$ & $\boldsymbol{\Lambda}$ & $\boldsymbol{\mu}$ & $\boldsymbol{\vartheta}$ _por \\
\hline $\boldsymbol{\rho}$ & $/$ & -0.81 & 0.76 & 0.48 & -0.92 \\
$\mathbf{C E}$ & -0.4 & $/$ & -0.55 & -0.32 & 0.81 \\
$\boldsymbol{\Lambda}$ & 0.97 & -0.36 & $/$ & 0.51 & -0.67 \\
$\boldsymbol{\mu}$ & 0.6 & 0 & 0.65 & $/$ & -0.59 \\
$\boldsymbol{\vartheta}$ _por & -0.93 & 0.43 & -0.96 & -0.59 & $/$
\end{tabular}

*Two correlation matrices are shown in one table: respectively above/below the symmetry axis the assumed correlation matrix for the brick/mortars is shown.

\subsection{Sample size}

Literature suggests diverse sample sizes. Lomas et al. [7] for example, suggests that in perspective of thermal modelling 60-80 simulations are sufficiently independent of the number of input parameters while Iman et al. [8] suggests a minimal sample size of $4 \mathrm{k} / 3$, with $\mathrm{k}$ the amount of input parameters. For 2D hygrothermal applications the necessary sample size typically increases. For this research the sample size is fixed to 258 as this is the minimal size to meet a

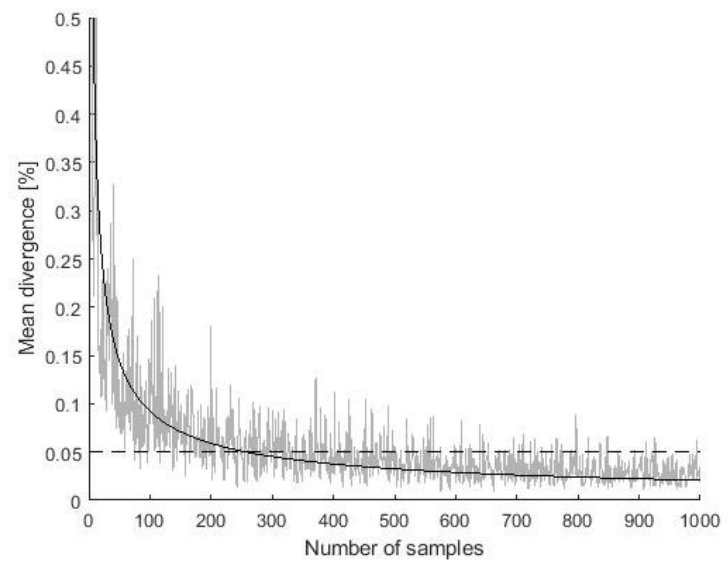
the correlation matrix of the uniform latin hypercube samples vs. the actual correlation matrix (Table 2.).

\section{Evaluation criteria for frost damage}

Due to the discussion about the relevance of frost criteria [1], [9] four criteria are used and compared. The first criterion is the maximum amount of critical freeze-thaw cycles (FTC) [-] with the critical moisture content equal to $25 \%$ of the effective moisture content 
(assumption based on Straube et al. [9]). The second, is the mean ice mass density(IMD) $\left[\mathrm{Kg} / \mathrm{m}^{3}\right]$ calculated with inclusion of the freezing point depression caused by the occurring capillary pressure. The third and fourth criteria are the moisture/temperature related stress of the construction between the exterior surface and a depth of $5 \mathrm{~mm} \mathrm{[3].} \mathrm{The}$ moisture/temperature related stress of the construction is a criterion that allows to evaluate the impact of thermal and hygric properties separately (Eq. 1 and 2).

$$
\begin{aligned}
\mathrm{HLD}_{T} & =\sum_{i=1}^{8760} \sqrt{\left(\bar{T}_{s, i}-\bar{T}_{5 m m, i}\right)+\Delta t_{i}{ }^{2}} \\
\mathrm{HLD}_{\vartheta} & =\sum_{i=1}^{8760} \sqrt{\left(\bar{\vartheta}_{s, i}-\bar{\vartheta}_{5 m m, i}\right)+\Delta t_{i}{ }^{2}}
\end{aligned}
$$

With $\bar{T}_{s, i}-\bar{T}_{5 m m, i}, \quad \bar{\vartheta}_{s, i}-\bar{\vartheta}_{5 m m, i}$ the mean temperature/relative humidity difference between the outer surface and at a depth of $5 \mathrm{~mm}$ in a time interval $\Delta \mathrm{t}_{i}(1$ hour $)$.

\section{Results and discussion}

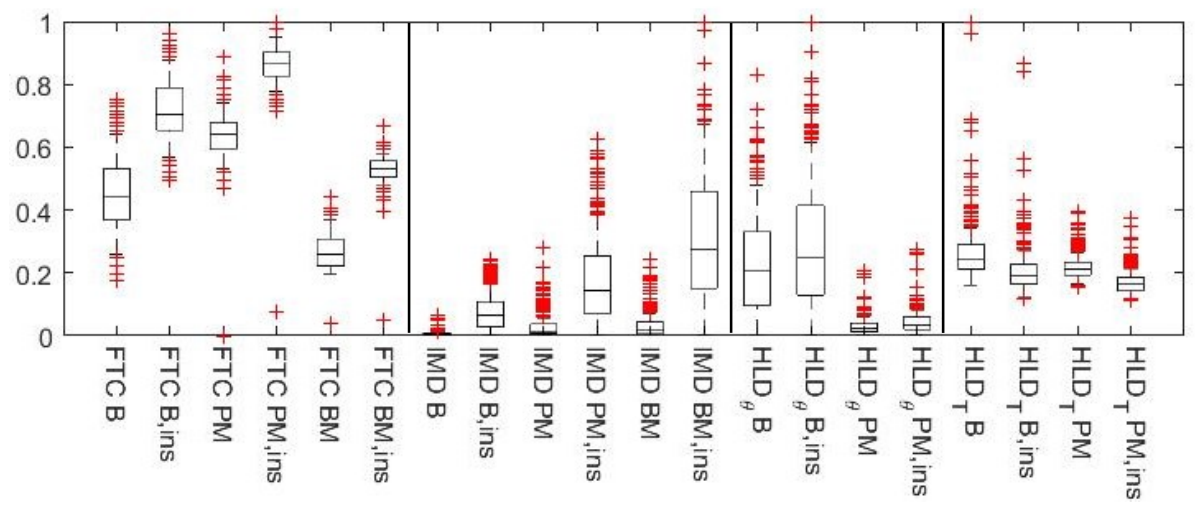

Fig. 3. Boxplot of the four frost damage criteria, the results are normalized for each criterion to ease comparison. The cross shaped markers indicate the outliner results which fall in the $5 \%$ tail of the distribution.

Figure 3 indicates that for each frost damage related criterion the results have a different mutual relationship. As expected, interior insulation increases the amount of FTC and the average IMD due to a decreased drying potential to the interior and a lowering of the overall temperature of the construction. The moisture related stress slightly increases as higher moisture contents are reached, while the thermal stress slightly decreases due to the more moderated temperature differences resulting from the insulation. Remarkable is that, while the relative differences based on FTC for each material between insulated and uninsulated are generally similar, this is not the case for the average IMD criterion. On the average IMD the impact increases from brick to pointing mortar to bed mortar. The main difference between the IMD and the FTC criterion is that a moisture content increase above

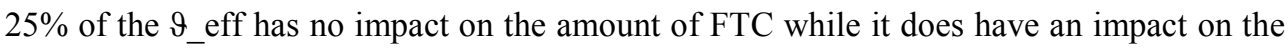
average IMD. Due to the reduced drying potential of the construction towards the inside, the bed mortar is only able to evacuate moist towards the pointing mortar. As the pointing mortar is located in front of the bed mortar the moisture storage of the pointing mortar $\vartheta \_$eff 
(PM) is a dominant impact factor over the IMD of the bed mortar. This is confirmed by Table 3 which ranks the impact parameters for each criterion based on a stepwise regression analysis. $\mathrm{R}_{\text {cum }}$ indicates how dependant the criterion is on all the, already mentioned, material properties in the ranking .

Table 3 Ranking of the input parameters by a stepwise regression analysis

\begin{tabular}{|c|c|c|c|c|c|c|}
\hline \multirow{2}{*}{$\begin{array}{c}\text { Rank } \\
\text { Criteria, Material, } \\
\text { (not) insulated }\end{array}$} & \multicolumn{2}{|c|}{ Rank 1} & \multicolumn{2}{|c|}{ Rank 2} & \multicolumn{2}{|c|}{ Rank 3} \\
\hline & Name & $R_{\text {cum }}^{2}$ & Name & $R_{\text {cum }}^{2}$ & Name & $R_{\text {cum }}^{2}$ \\
\hline FTC, $B$, ins. & $\vartheta \_$eff $(B)$ & 0.351 & $\vartheta \_$eff (PM) & 0.530 & KI_eff (BM) & 0.543 \\
\hline$F T C, B$, not ins. & $\vartheta \_$eff $(B)$ & 0.297 & $\lambda(B)$ & 0.465 & $\vartheta \_$eff $(P M)$ & 0.598 \\
\hline$F T C, P M$, ins & $\lambda(B M)$ & 0.094 & KI_eff $(B)$ & 0.169 & KI_eff (BM) & 0.236 \\
\hline$F T C, P M$, not ins. & $\lambda(B)$ & 0.222 & $\lambda(B M)$ & 0.417 & KI_eff $(B)$ & 0.485 \\
\hline$F T C, B M$, ins. (22) & KI_eff(B) & 0.131 & $\lambda(B M)$ & 0.189 & $\lambda(B)$ & 0.225 \\
\hline FTC, BM, not ins. & $\lambda(P M)$ & 0.373 & $\lambda(B M)$ & 0.503 & $\lambda(B)$ & 0.583 \\
\hline$I M D, B$, ins. & $\vartheta \_$eff $(B)$ & 0.431 & $K I \_$eff $(P M)$ & 0.526 & $\lambda(B)$ & 0.568 \\
\hline$I M D, B$, not ins. & $\vartheta_{-}$eff $(B)$ & 0.399 & KI_eff (B) & 0.453 & $\lambda(B)$ & 0.501 \\
\hline IMD, PM, ins. & $\vartheta \_$eff(BM) & 0.785 & $K I \_$eff $(B M)$ & 0.799 & $K I \_e f f(P M)$ & 0.813 \\
\hline$I M D, P M$, not ins. & $\vartheta \_$eff (BM) & 0.409 & $\lambda(B)$ & 0.461 & KI_eff (B) & 0.513 \\
\hline$I M D, B M$, ins. & $\vartheta \_$eff (PM) & 0.712 & $\lambda(B)$ & 0.721 & KI_eff (PM) & 0.731 \\
\hline$I M D, B M$, not ins. & $\vartheta \_$eff $(P M)$ & 0.338 & KI_eff $(B)$ & 0.430 & $\vartheta \_$eff $(B)$ & 0.457 \\
\hline$H L D_{T}, B$, ins. & $\lambda(B M)$ & 0.536 & $\vartheta \_$eff (BM) & 0.564 & $\vartheta \_e f f(B)$ & 0.576 \\
\hline$H L D_{T}, B$, not ins. & $\lambda(B M)$ & 0.587 & $\vartheta \_$eff (BM) & 0.610 & $\lambda(B)$ & 0.619 \\
\hline$H L D_{T}, P M$, ins. & $\lambda(B)$ & 0.816 & $\vartheta \_$por (BM) & 0.836 & $R O(B)$ & 0.843 \\
\hline$H L D_{T}, P M$, not ins. & $\lambda(B)$ & 0.805 & $\lambda(B M)$ & 0.836 & $\lambda(P M)$ & 0.874 \\
\hline$H L D_{\vartheta}, B$, ins. & $\vartheta \_$eff $(B M)$ & 0.869 & KI_eff (BM) & 0.937 & KI_eff $(B)$ & 0.948 \\
\hline$H L D_{\vartheta}, B$, not ins. & $\vartheta \_$eff $(B M)$ & 0.900 & KI_eff (BM) & 0.937 & KI_eff $(B)$ & 0.947 \\
\hline$H L D_{\vartheta}, P M$, ins. & $\vartheta \_$eff $(B)$ & 0.300 & KI_eff $(B)$ & 0.737 & $\lambda(B)$ & 0.744 \\
\hline$H L D_{\vartheta}, P M$, not ins. & $\vartheta \_$eff $(B)$ & 0.308 & KI_eff $(B)$ & 0.710 & $\vartheta \_p o r(B M)$ & 0.718 \\
\hline
\end{tabular}

Note that $\vartheta_{-}$eff (PM), $\vartheta_{\text {eff }}(\mathrm{BM})$ are dominant over each other's IMD as the same course of the moisture retention curve is assumed which makes that changes on the effective saturation lead to a redistribution of moist in between these two materials and as a result especially the IMD frost criterion is influenced. For IMD in the pointing mortar the effect of the adding of insulation is less pronounced than for the brick and is pointing to the exterior surface due to its proximity.

In reality this effect is often found when the properties of the pointing mortar are not in tune with the bed mortar, freezing behind the pointing mortar occurs and the pointing mortar is pushed out of the joint.

For the FTC criterion it is clear based on Table 3 that the effective saturation is less dominant than for the IMD. Here no clear material property dominates as all $\mathrm{R}_{\text {cum }}$ values are quite low. 
The $\mathrm{HLD}_{\mathrm{T}}$ criterion is mainly dominated by the thermal conductivity of the adjacent material as this material will mute or strengthen the thermal shocks. A similar effect is seen for the $\mathrm{HLD}_{9}$ criterion, with the effective saturation. The second most important parameter is the liquid conductivity of the adjacent materials as the speed of moisture redistribution is also important for the occurrence of hygric shocks.

Table 3 indicates in general that the most important impact parameters are respectively the effective moisture content, the liquid conductivity and the thermal conductivity of the materials. Note as well that often the properties of the material itself are less dominant than the properties of the adjacent material in this construction. For example, the $\mathrm{HLD}_{\mathrm{T}}$ criterion of the pointing mortar depends more on the lambda value of the adjacent brick and bed mortar than of the lambda value of the pointing mortar itself. This could be explained by the small size of the pointing mortar, but then again for the $\mathrm{HLD}_{\mathrm{T}}$ criterion of the brick, the lambda value of the bed mortar has a bigger impact than the lambda value of the brick itself. This indicates the complex interaction between the hygrothermal and geometric aspects within the masonry construction.

\section{Conclusion}

Despite the high uncertainty of the material properties of bricks and mortars it is possible to subtract the main impact material parameters for a specific construction based on probabilistic Heat, Air and Moisture simulations although the chosen damage criteria has a significant impact. In this paper a 2D-section of masonry is simulated with stochastic material property input data, and the dominant input parameters related to several frost damage criteria are analysed and ranked. The often returning impact parameter in perspective to the frost damage is the effective moisture content together with the liquid and thermal transport properties of each material in the construction. The risk of frost damage on a certain location is often dominated by the properties of an adjacent material, which emphasizes that measurements on these properties for each material are a prerequisite before starting a renovation or restoration as well as for repointing insulation measures.

\section{References}

1. T. De Kock, D. Guilbert, S. Caluwaerts, K. Calle, N. Van Den Bossche, V. Cnudde, Sci. Total Environ., pp. 1-17, (To be published)

2. CEN, EN ISO $13788,(2001)$

3. J. Zhao, Mech. Aerosp. Eng. - Diss., (2012)

4. J. G. Carl-Eric Hagentoft, N. M. M. Ramos, J. Grunewald, Annex 55: Stochastic Data, (2015)

5. H. Janssen, Reliab. Eng. Syst. Saf., vol. 109, pp. 123-132 (2013)

6. E. Vereecken, L. Van Gelder, H. Janssen, S. Roels, Energy Build., vol. 89, pp. 231244, (2015)

7. K. J. Lomas, H. Eppel, Energy Build. 19 21-44, vol. 19, pp. 21-44, (1992)

8. J. C. Iman, R.L. Helton, 'A comparison of uncertainty and sensitivity techniques ...' (1985)

9. J. Straube, C. Schumacher, P. Mensinga, Proc. Performances Envel. Whole Build. XI, pp. 1-8, (2010) 